\title{
Numerical study of a banded precipitation event over Italy
}

\author{
P. Malguzzi, M. Fantini, and A. Buzzi \\ ISAC-CNR, via Gobetti 101, 40129 Bologna, Italy
}

Received: 15 February 2010 - Revised: 24 May 2010 - Accepted: 25 May 2010 - Published: 30 June 2010

\begin{abstract}
Satellite images of 30 October 2008 show the development over north-central Italy of rainbands and multiple waves during a strong south-westerly wind episode associated with a deepening synoptic trough and cold front passage. The event was studied by means of the ISAC model chain constituted of the hydrostatic model BOLAM and the nested non-hydrostatic model MOLOCH at $1.1 \mathrm{~km}$ resolution. Diagnostics of model output was performed to reveal the physical origin of the dynamical features and precipitation field as simulated. Based on our results we propose a theoretical framework in which symmetric instability underlies some of the observed precipitation patterns.
\end{abstract}

\section{Introduction}

MSG Satellite images of 30 October 2008 (Fig. 1) show the development over North-Central Italy of several rainbands and multiple wavetrains during a strong south-westerly wind episode associated with a deepening synoptic trough and cold front passage. The nearly two-dimensional structure of the large scale flow (Fig. 2) and the ubiquitous orographic forcing makes this a nearly ideal setup to test for the occurrence of slanted circulations developing in a symmetrically unstable environment.

For this purpose we used the ISAC model chain, constituted of the hydrostatic model BOLAM and the nonhydrostatic model MOLOCH, to simulate the episode, and performed diagnostics that support the hypothesis that symmetric instability was present during the event.

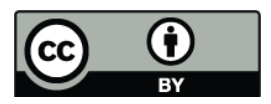

Correspondence to: M. Fantini (m.fantini@isac.cnr.it)

\section{The non-hydrostatic model MOLOCH}

The non-hydrostatic model MOLOCH integrates the fully compressible set of equations, using as prognostic variables pressure, temperature, specific humidity, horizontal and vertical velocity components, and five condensed water species. Model dynamics are integrated in time with an implicit scheme for the vertical propagation of sound waves, while explicit, time-split schemes are implemented for the remaining terms. Advection is computed using the Eulerian WAF scheme (Billet and Toro, 1997). The vertical grid is stretched exponentially with height. The microphysical scheme is based on the parameterization proposed by Drofa and Malguzzi (2004). The physical processes determining the time tendency of specific humidity, cloud water/ice and precipitating water/ice are divided into "fast" and "slow" ones. Fast processes involve transformations between specific humidity and cloud quantities, while slow ones involve rain/snow/hail production and fall. Temperature is updated by imposing exact enthalpy conservation at constant pressure. Fall of precipitation is computed with the stable and dispersive backwardupstream scheme, with speed depending on concentration. For a full presentation of the model see Malguzzi et al. (2006), which also contains a description of BOLAM.

\section{Simulation}

BOLAM was run for $15 \mathrm{~h}$, starting from NCEP analysis at 00:00 UT with boundary conditions every three hours taken from the NCEP global daily forecast, with horizontal resolution $13 \mathrm{~km}$ and 40 vertical levels. The non-hydrostatic MOLOCH was then nested in the BOLAM domain, starting at 01:00 UT, with boundary conditions updated at $30 \mathrm{~min}$ interval. MOLOCH was run at 0.01 degrees resolution, corresponding to $1.1 \mathrm{~km}$, and 50 vertical levels, on the domain shown in Fig. 3. 


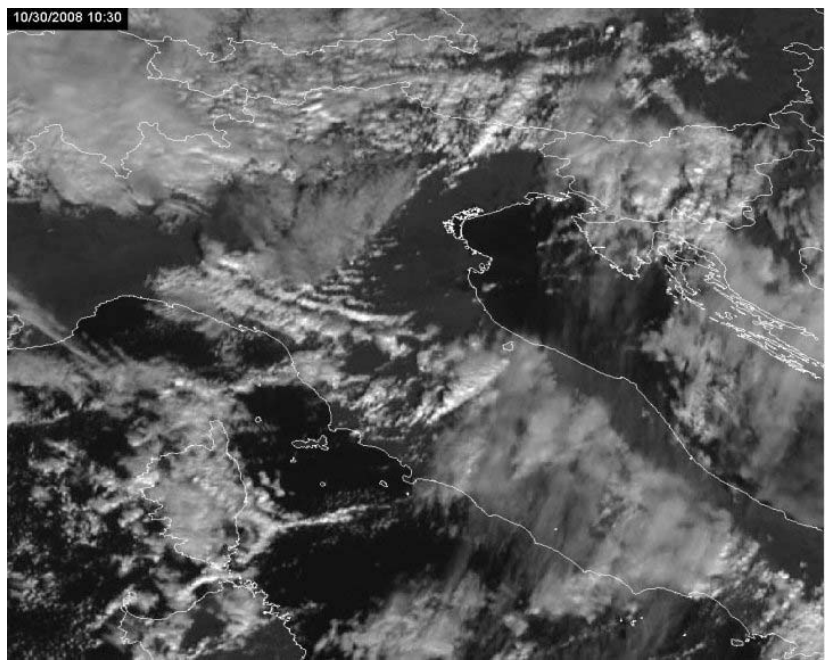

Fig. 1. Satellite image at 10:30 UT 30 October 2008 showing wave trains in the lee of the Northern Apennines mountains and several rainbands at various stages of development. Image Copyright CC2008 EUMETSAT, courtesy V. Levizzani, ISAC-CNR.

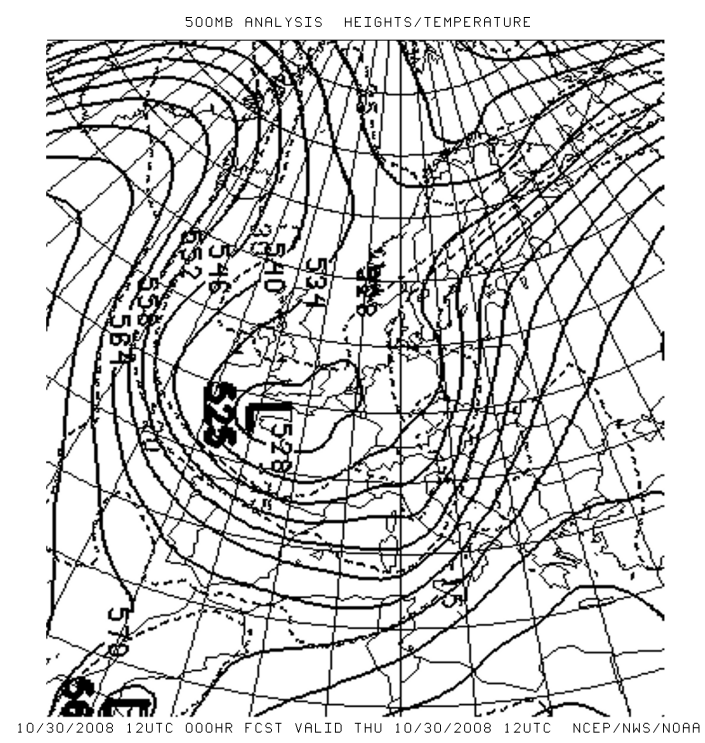

Fig. 2. $500 \mathrm{hPa}$ analysis (height and temperature) at 12:00 UTC 30 October 2008. Modified from NWS DIFAX Weather Map Archive at http://archive.atmos.colostate.edu/.

The MOLOCH simulation generates deep convective activity from early morning to about 12:00 UTC, mainly in the south-east part of the domain, in the warm and moist sector ahead of the front.

Between 09:00 and 10:30 slanted convective lines appear over Central Italy, oriented SW to NE. These lines are aligned with the jet, perpendicular to the Apennines and well distinct from the lee waves that can be observed, in Fig. 1,

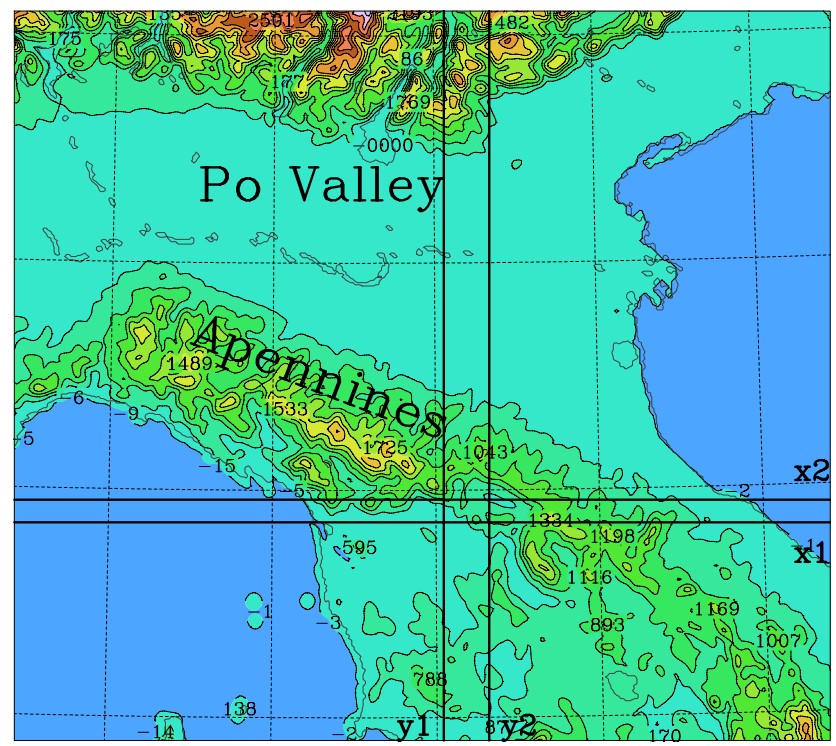

Fig. 3. MOLOCH domain of integration, topography and land-sea mask. Contour interval $250 \mathrm{~m}$. The thick straight lines identify the location of the cross-sections shown in Figs. 9-12.

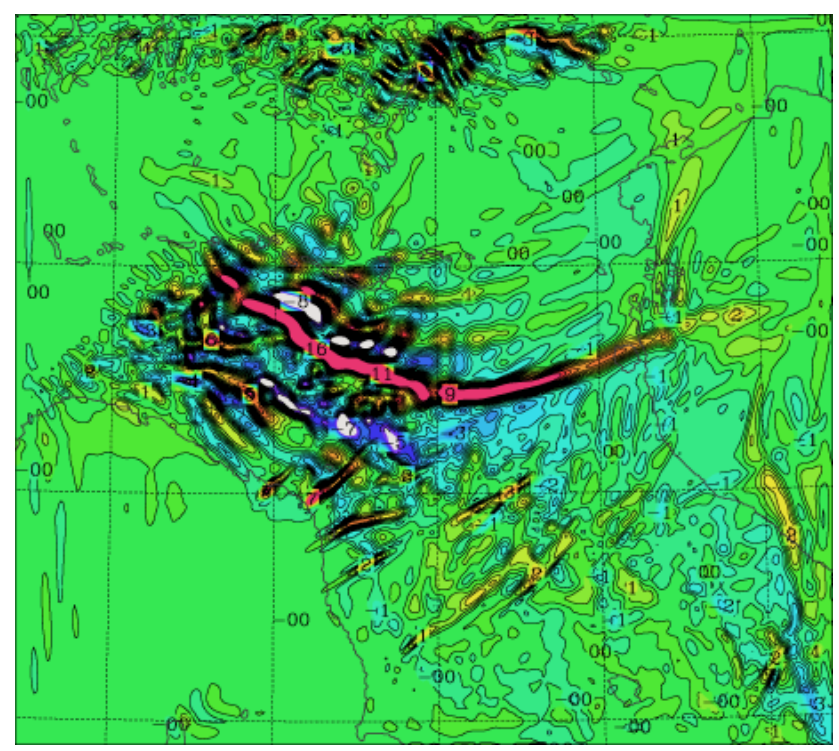

Fig. 4. Vertical velocity at $700 \mathrm{hPa}, 09: 30 \mathrm{UTC}$ from MOLOCH simulation. Contour interval $0.5 \mathrm{~ms}^{-1}$.

over the Po Valley. From these simulations we show vertical velocity $w$ at $700 \mathrm{hPa}$ (Fig. 4), accumulated precipitation in $30 \mathrm{~min}$ (Fig. 5) and wind vectors at two levels (Figs. 6 and 7). A strong downslope wind started early in the morning in the lee of the Northern Apennines, reaching maximum amplitude at 09:00 UT, when the simulated $10 \mathrm{~m}$ wind reaches maxima of $30 \mathrm{~m} / \mathrm{s}$. Damages due to strong gustiness were reported in several mountain locations during the late morning 


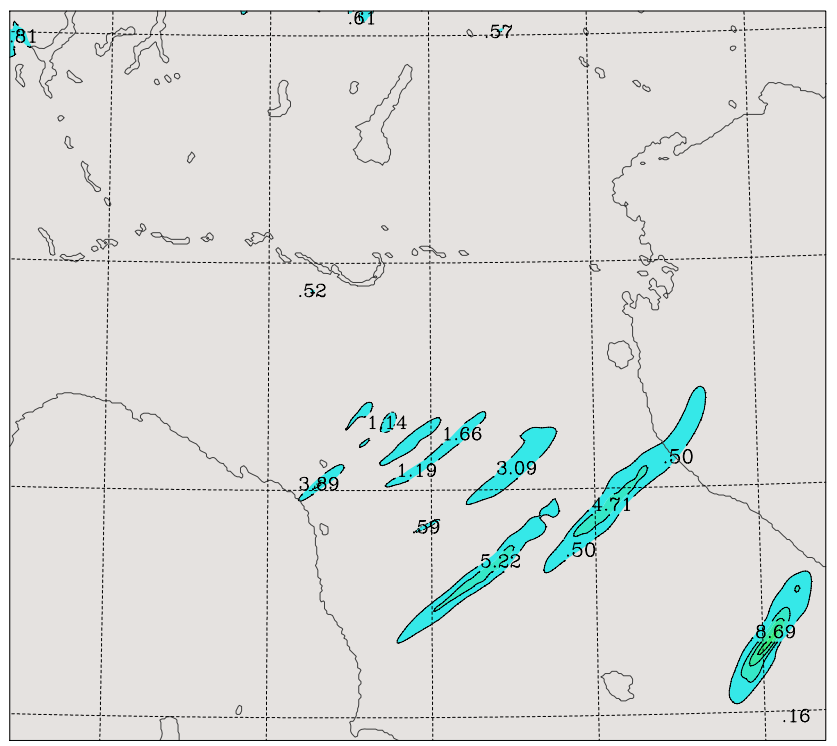

Fig. 5. Accumulated precipitation between 09:00 and 09:30 UTC. Contour interval $2.5 \mathrm{~mm}$, starting from 0.5 to remove the noisy zero contour.

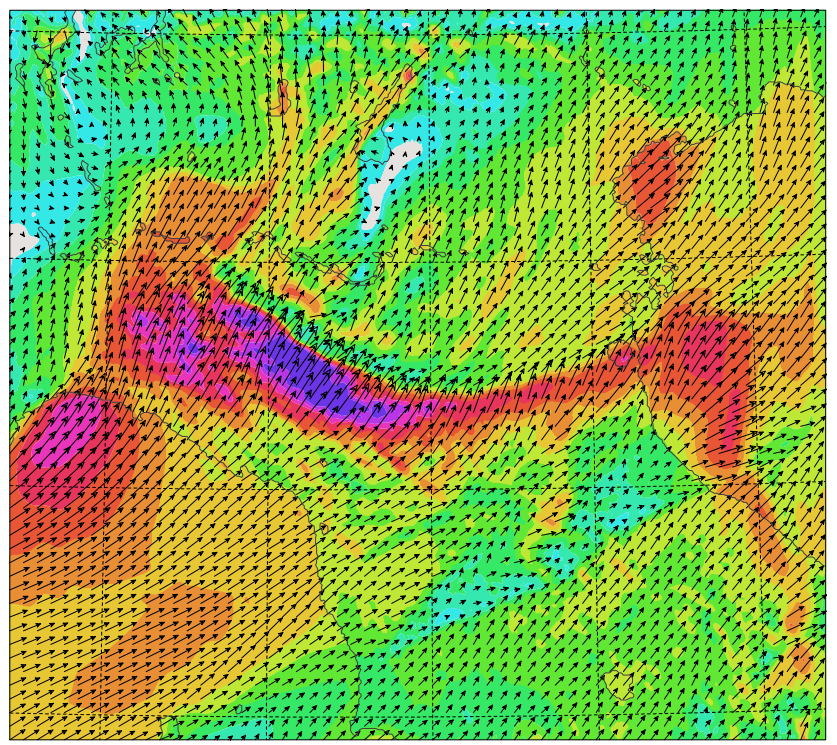

Fig. 6. Wind vectors at $10 \mathrm{~m}$ at 09:30 UT, with contours of wind speed, interval $2 \mathrm{~m} \mathrm{~s}^{-1}$.

hours. The MOLOCH simulation reproduces well the intensity of this wind, as seen in Figs. 6 and 7.

The waves over the Po Valley are orographically generated, have maximum amplitude near the ground and displace too little moisture to form any precipitation. The cloud bands over Central Italy are convectively active, with maximum intensity in the mid troposphere, and are precipitating. We also show (Fig. 8) the simulated relative humidity at $600 \mathrm{hPa}$ that

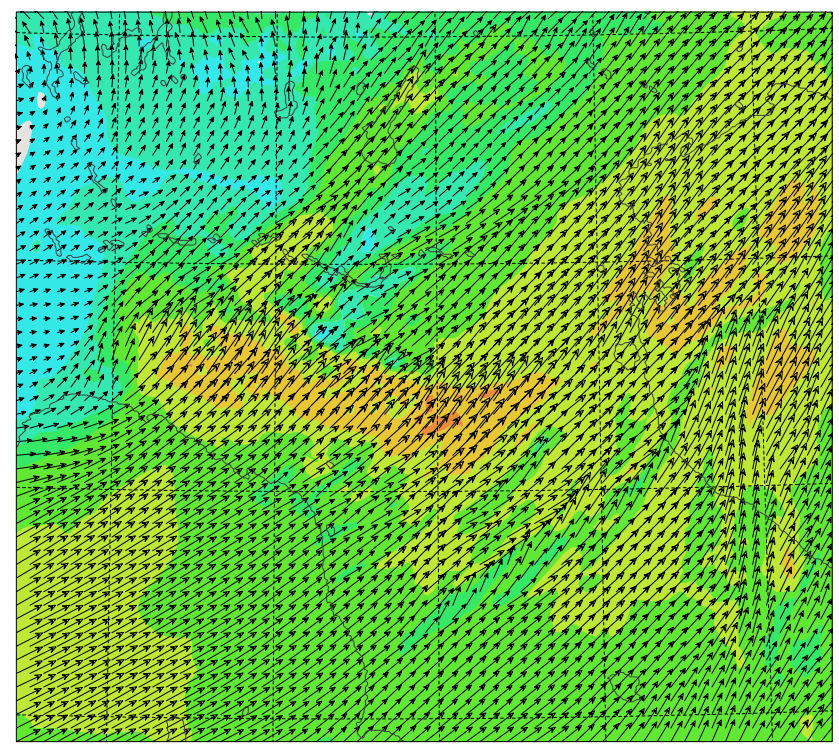

Fig. 7. Same as Fig. 6 except at $700 \mathrm{hPa}$. Contour interval $5 \mathrm{~m} \mathrm{~s}^{-1}$.

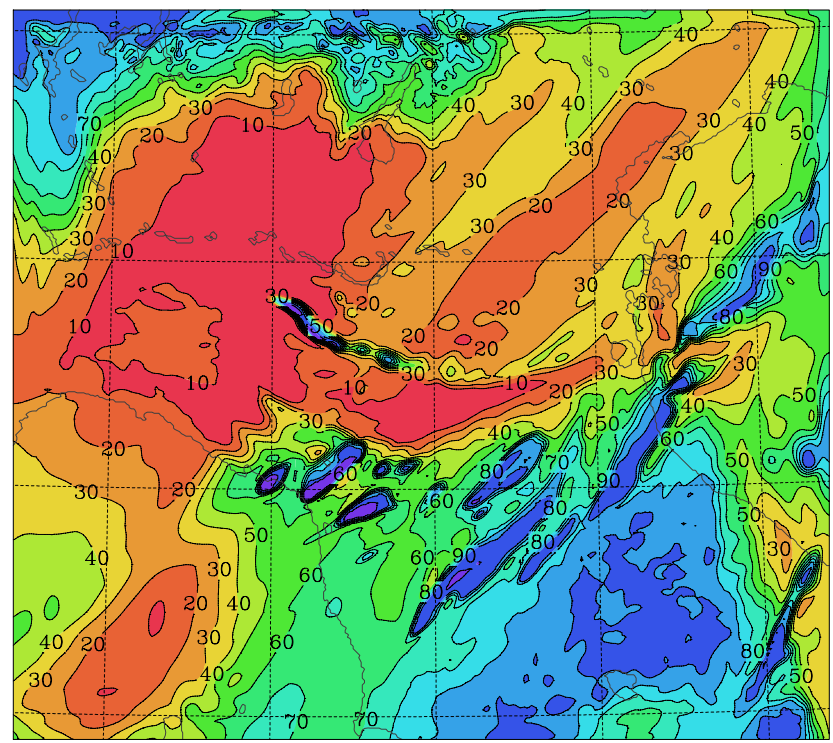

Fig. 8. Relative humidity at $600 \mathrm{hPa}, 09: 30 \mathrm{UTC}$. Contour interval 10 percent.

exhibits a rather sharp boundary between the moist warm area ahead of the front and the dryer air behind it.

Figures 9, 10 and 11 present cross-sections of relative humidity (colours) and cloud condensate (water+ice, contours) taken along the sections identified by thick straight lines in Fig. 3. Several episodes of slantwise convection at various stages of evolution can be seen, with cloud top reaching $7000 \mathrm{~m}$ at most. Cells arising from more traditional 


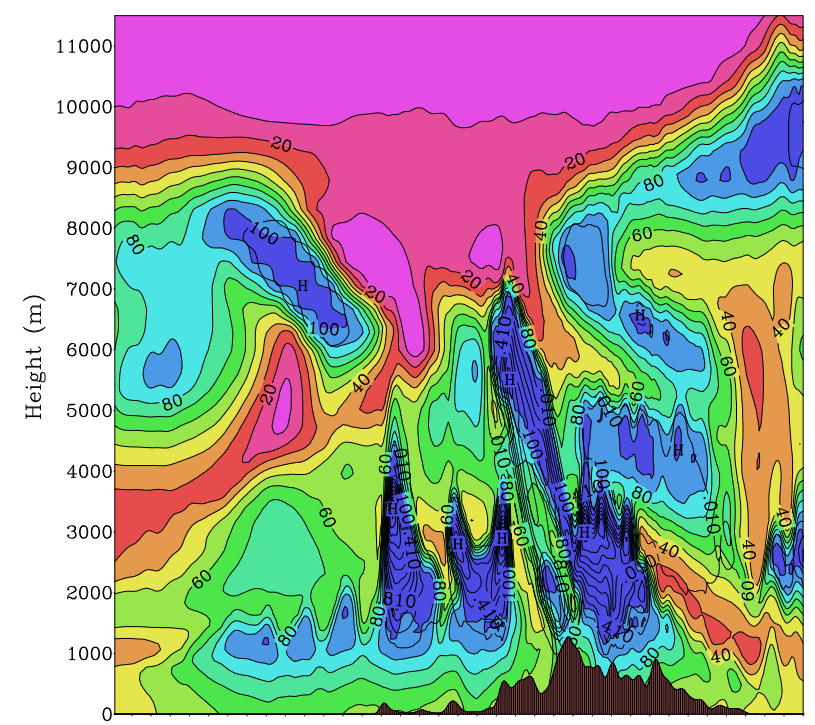

Fig. 9. Relative humidity (color) and water condensate (thick contours) at 09:30 UTC on section x1 (see Fig. 3).

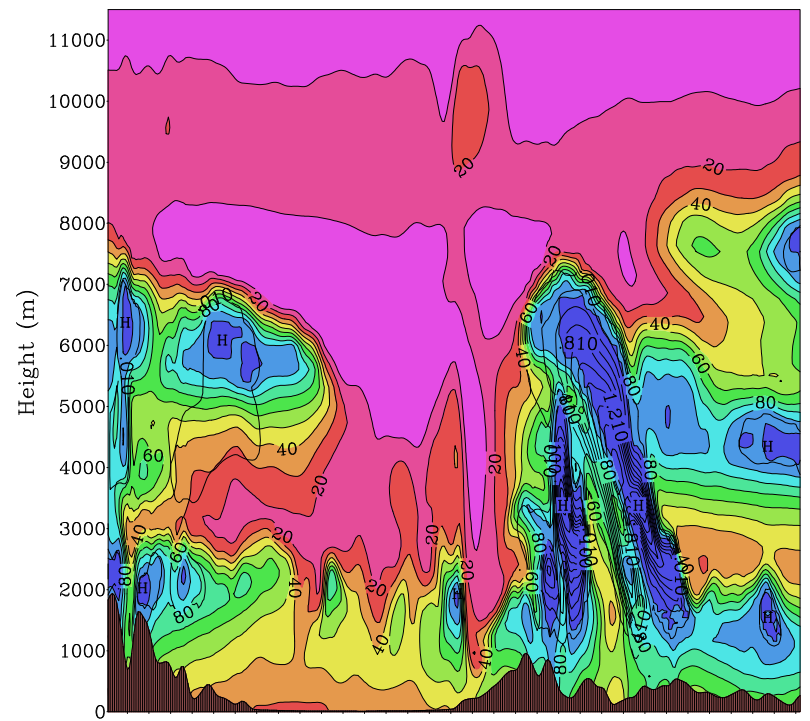

Fig. 10. Same as Fig. 9 on section y2 at 09:30 UT.

convective instability, due to moist unstable conditions at low levels, are also present in the simulation. These cells tend to develop into mainly shallow convection, which coexists with slantwise convection. The last figure (Fig. 12) shows a typical cross-section of saturated equivalent potential temperature. Unstable conditions are indeed observed in the layer below $2000 \mathrm{~m}$. Solid contours in the same figure are pseudo angular momentum ( $M$, see Appendix A) iso-surfaces computed with the wind across the section. Where slanted convection originates and develops, isolines

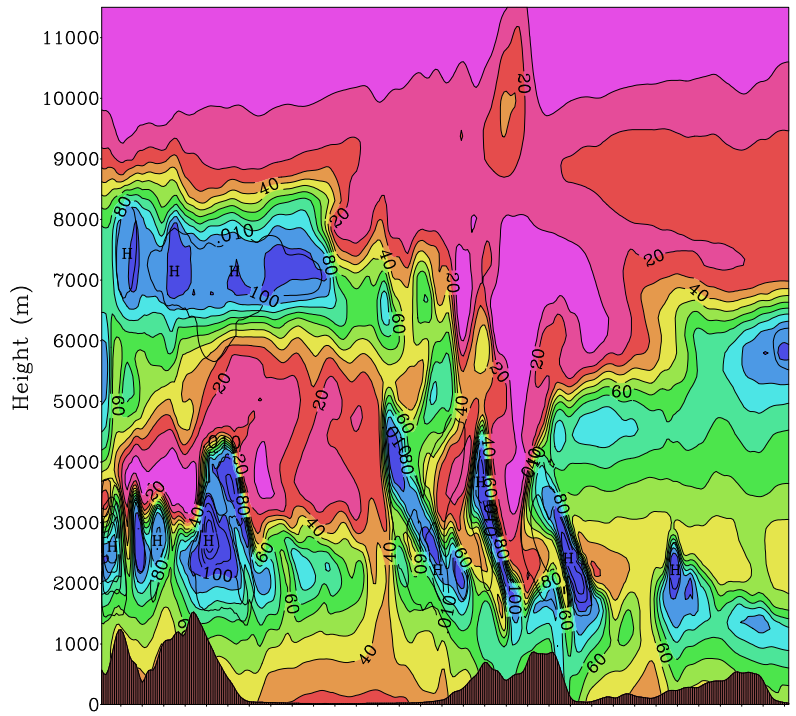

Fig. 11. Same as Fig. 9 on section y1 at 10:30 UTC.

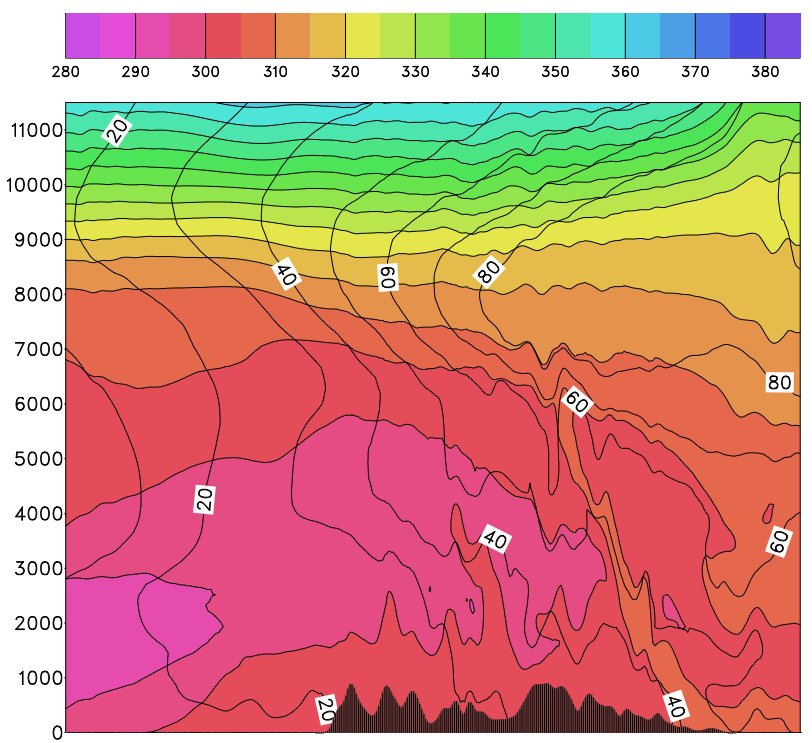

Fig. 12. Saturated equivalent potential temperature $\theta_{e}$ (color) and pseudo angular momentum $M=v+f x$ (thick contours) at 09:00 UTC on section $\mathrm{x} 2$ of Fig. 3 (contour interval $10 \mathrm{~m} \mathrm{~s}^{-1}$, arbitrary origin).

of equivalent potential temperature and $M$ tend to be, on average, aligned, realizing the condition for marginal moist symmetric instability. 


\section{Conclusions}

We do not consider this evidence conclusive. However, the growth and permanence, for times of the order of one hour, of narrow regions of saturated, precipitating, slanted ascent across the wind (NOT differential advection) and in a direction consistent with the wedge of symmetric instability, and the formation of persistent neutral channels as the end result of the slanted ascent (slantwise convective adjustment) are strong indications that symmetric instability may have taken place in this event. The coexistence of conditions leading to moist convection and symmetric instability, as well as the complexity introduced by orography, the threedimensionality and non-stationarity of the large scale flow make it difficult to isolate the signature of moist symmetric instability in this environment. Numerical simulations in more idealized and controllable conditions should be the next step in the process to understand the role played by symmetric instability in determining frontal structure.

\section{Appendix A}

\section{Symmetric instability}

For a nearly parallel flow one can look at a representative section across the flow and consider the two dimensional problem. Define $M=f x+v$ and let $B=\theta$ in dry conditions, or $B=\theta_{e}$ for saturated flow. Then $M_{x}<0$ denotes inertial instability and $B_{z}<0$ denotes convective instability. When the derivatives are positive but the Jacobian $Q$ is negative:

$Q=\frac{\partial(M, B)}{\partial(x, z)}=M_{x} B_{z}-M_{z} B_{x}<0$ then unstable slanted updrafts are still possible. As is the case with ordinary convection, if the air is sub-saturated the instability is "conditional", i.e. external forcing is required to kick-start the motion. Moreover, the consideration of condensate loading changes somewhat the above condition (Fantini and Malguzzi, 2008).

This instability favors structures elongated along the wind and it is therefore generally invoked as a possible explanation for frontal rain-bands (Bennetts and Hoskins, 1979). The quantity $Q$ above (a two-dimensional potential vorticity) can be interpreted as the gradient of $M$ along the surfaces of constant $B$, or the gradient of $B$ along $M$ surfaces (with appropriate changes of sign). The state of neutrality $Q=0$, easily identified as the two family of isopleths of $B$ and $M$ become parallel, presumably as the final outcome of the instability, is usually referred to as "slantwise convective adjustment".

Edited by: G. Boni

Reviewed by: one anonymous referee

\section{References}

Bennetts, D. A. and Hoskins, B. J.: Conditional Symmetric Instability - A Possible Explanation for Frontal Rainbands, Q. J. Roy. Meteor. Soc., 105, 945-962, 1979.

Billet, S. and Toro, E. F.: On WAF-type schemes for multidimensional hyperbolic conservation laws, J. Comput. Phys., 130, 1 24, 1997.

Drofa, O. V. and Malguzzi, P.: Parameterisation of microphysical processes in a non-hydrostatic prediction model, in: Proceedings of 14th Intern. Conf. on Clouds and Precipitation (ICCP), Bologna, Italy, 19-23 July 2004, 1297-1300, 2004.

Fantini, M. and Malguzzi, P.: The slope of moist symmetric instability with water loading, J. Atmos. Sci., 65, 2922-2935, 2008.

Malguzzi, P., Grossi G., Buzzi A., Ranzi R. and Buizza R.: The 1966 'century' flood in Italy: A meteorological and hydrological revisitation, J. Geophys. Res., 111, D24106, doi:10.1029/2006JD007111, 2006. 